\title{
Evaluation of Practical Process Aspects for Lipozyme TL IM Catalyzed Bulk Fat Modification in a Batch Reactor
}

\author{
Hong Zhang*
}

BioCentrum-DTU, Technical University of Denmark, DK-2800 Lyngby, Denmark

\begin{abstract}
A few issues to apply Lipozyme TL IM-catalyzed interesterification for bulk fat modification were investigated in a batch reactor system with concerning practical process development. The hydrolyzed products, i.e. free fatty acids and diglycerides, generated from the Lipozyme TL IM-catalyzed interesterification process due to water participation, can be minimized by enzyme pre-treatment. For the interesterified products, free fatty acids formed by hydrolysis had softening effect on solid fat content and diglycerides retarded crystal transformation during storage. Reaction time was more critical for the reduction of acyl migration than the control of water content in the system. The temperature effect on the rate of interesterification in terms of $\mathrm{Q}_{10}$ value was determined as 1.3, meaning that the increase of reaction rate was limited by the increase of temperature. Therefore, for practical implementations, a temperature of $60-70{ }^{\circ} \mathrm{C}$ which the feed stock can be totally melted for the reaction should be used in consideration of enzyme stability. The quality of feedstock was confirmed to be critical to maintain the enzyme activity and the refined, bleached, and deodorized oil should be used as the feedstock.
\end{abstract}

Key Words: Enzymatic interesterification, Lipozyme TL IM, batch reactor, by-products, oil quality.

\section{INTRODUCTION}

With the development and commercialization of Lipozyme TL IM, a silica granulated Thermomyces lanuginosus lipase, the push for the uses in bulk fat modification to replace the chemical interesterification has been intensified [1-12]. The lipase was initially developed for detergent uses [1], but it was successfully demonstrated for the modification of bulk fat after further development [2]. The reaction behavior was widely studied in batch as well as continuous reactors $[2,8,11]$. Thermomyces lanuginosus lipase has been generally regarded as regio-specific for sn-1,3 positions. However, the specificity can be influenced by many issues such as media, substrates, and even acyl migration [2,7]. The reaction kinetics with conversion defined by solid fat content was also demonstrated as a useful monitoring index for process control [3]. The possibility of using FTIR or FTNIR for online monitoring of the Lipozyme TL IM- catalyzed interesterification process was further demonstrated and proved to be feasible $[9,10]$. The physical properties as well as storage properties of Lipozyme TL IM-produced fats or fat products were also systematically investigated [4-6,12]. Commercial sectors have also made strong efforts to promote, engage or implement the technology for the upgrading of traditional processing technology (www.novozymes.com, www.desmetgroup.com, www.admworld.com, etc.).

For the operation of the technology in actual uses, there certainly remain a number of issues that are needed to be solved. This is certainly true for a technology developed only for fewer than a dozen of years. For an immediate practical thinking, there are a few issues remaining hanging around in

\footnotetext{
*Address correspondence to this author at the BioCentrum-DTU, Building 222, Technical University of Denmark, DK-2800 Lyngby, Denmark; Tel: +45 4525 2637; Fax: +45 4588 4922; E-mail. hz@ biocentrum.dtu.dk
}

the innovation path, such as how the water in the commercial lipase preparation affects the processing in terms of byproducts and enzyme activity, how those byproducts affect the physical properties of the products, how critically reaction can be benefited through temperature changes, and how substrate material quality affects the enzyme activity, etc. Therefore, these issues were subjected to a critical evaluation in this study. We expect that the results should be useful for those practical users of the industrial sectors.

\section{MATERIALS AND METHODOLOGY}

\section{Materials}

Lipozyme TL IM, a commercial silica granulated Thermomyces lanuginosus lipase preparation (Novozymes A/S, Bagsvaerd, Denmark), was used for the lipase-catalyzed interesterification. Particle size was measured as 250 to 1000 $\mu \mathrm{m}$ (Fig. 1). Bulk density was $420 \mathrm{~kg} / \mathrm{m}^{3}$ and true density was $1830 \mathrm{~kg} / \mathrm{m}^{3}$. Soybean oils (SO, non-deodorized and deodorized) were supplied by Oelmühle Leer Connemann (Hamburg, Germany). Palm stearin (PS), coconut oil (CO), and rapeseed oil (RO) were supplied by AArhusKarlshamn (Karlshamns, Sweden). Fully hydrogenated cottonseed oil (FHCO) was supplied by Bunge Oils, Inc. (St. Louis, MO). L- $\alpha$ - phosphatidylcholine (PC, purity 95\%) was purchased from Sigma (St. Louis, MO). All other chemicals and reagents for the analysis were of analytical or chromatography grade.

\section{Batch Reaction}

Experiment was carried out in a solvent-free system by using $600 \mathrm{~g}$ oil blend with the lipase dosage of $10 \%$ at a defined reaction temperature. Interesterified products were collected from the Lipozyme TL IM-catalyzed interesterification at the different reaction times and stored in freezer 
before analysis. Lipozyme TL IM was used for the reaction either as it was or a pre-treatment was made as follows. Lipozyme TL IM contains appox. $5 \mathrm{wt} \%$ equilibrium water including 2 wt $\%$ bounded water [14]. The non-bound free water will then lead to a high amount of by-products formed by hydrolysis reaction during the interesterification process. The free water can be reduced by pre-treating the lipase with initial three reaction batches using $\mathrm{RO}$ or $\mathrm{SO}(600 \mathrm{~g})$ in 30 $\mathrm{min}$ at $70{ }^{\circ} \mathrm{C}$. After $30 \mathrm{~min}$ of incubation for each batch, the oil was removed through an in-situ filter. Finally the lipase was further quickly washed one more time with the designed oil blend before actual experiments. By this washing, the free water content was removed.

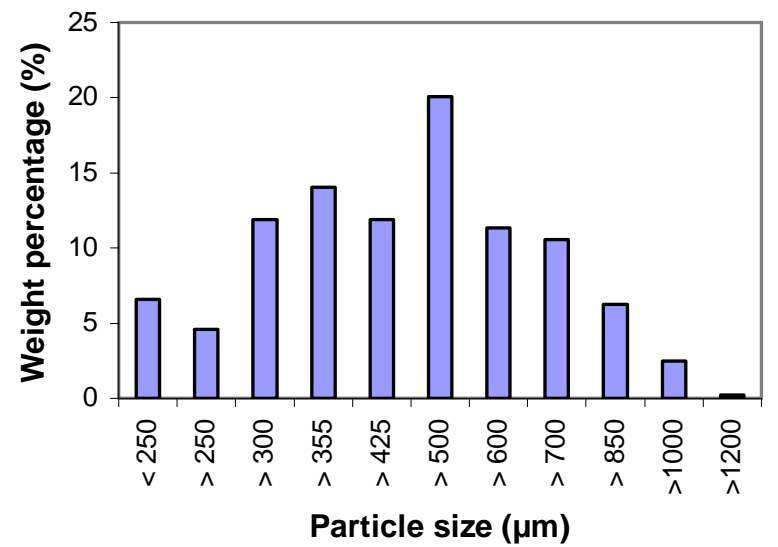

Fig. (1). Particle size distribution of Lipozyme TL IM.

\section{Temperature Effect on Reaction Rate}

The reaction rate was measured based on the changes of solid fat content with the choice of $40{ }^{\circ} \mathrm{C}$ and in $2 \mathrm{~h}$ reaction time using the blend of PS/CO (70/30) with lipase dosage of 4 wt\% Lipozyme TL IM.

\section{Short Path Distillation (SPD) to Remove Free Fatty Acids (FFA)}

A UIC KD-4 system (Alzenau-Hoerstein, Germany) was used to remove FFA from the products. Feeding temperature was set at $75{ }^{\circ} \mathrm{C}$. Evaporator and condenser temperatures were 190 and $75^{\circ} \mathrm{C}$, respectively. Feeding rate was in a range of 200-300 mL/h. Samples were distilled twice in order to reach a low content of FFA.

\section{Silica Gel Absorption to Remove Diglycerides (DAG)}

A simple absorption procedure was used to remove DAG as described by Reddy and Prabhakar [13].

\section{Triglyceride Profiles}

Triglycerides (TAG) were analyzed by reversed-phase HPLC. Separation was performed on a LiChroCART 250-4 (RP C18 end-capped) column (particle size $=5 \mu \mathrm{m}$, Merck, 64271 Darmstadt, Germany) with a binary solvent system of acetonitrile (solvent A) and dichloromethane (solvent B). Solvent B was increased from 25 to $27 \%$ over $20 \mathrm{~min}$, and followed by $100 \%$ solvent B for 5 min and then followed by the initial binary solvent for the equilibration of the system. Sample $(20 \mu \mathrm{l})$ was dissolved in $1 \mathrm{~mL}$ chloroform and $10 \mu \mathrm{l}$ aliquots were injected. Two typical peaks which changed mostly during reaction at retention time of $14.5\left(\mathrm{P}_{\mathrm{a}}\right)$ and 15.8 $\left(\mathrm{P}_{\mathrm{b}}\right)$ min were chosen to monitor the process.

$$
\text { Peak ratio }=\frac{\text { Area of peak } P_{a}}{\text { Area of peak } P_{b}}
$$

\section{Conversion Degree of Lipase-Catalyzed Interesterifica- tion}

The conversion degree $(\mathrm{X})$ is defined as:

$$
X(\%)=\frac{P_{t}-P_{o}}{P_{\infty}-P_{o}} \times 100
$$

where $P_{t}$ is the peak ratio at time $t, P_{\infty}$ is the peak ratio at equilibrium and $\mathrm{P}_{\mathrm{o}}$ is the peak ratio at time 0 . During the enzymatic interesterification, conversion degree can be controlled by changes of the reaction time.

\section{Fatty Acid Composition at the sn-2 Position}

The Grignard degradation method was used as described before [2].

\section{Fatty Acid Compositions (FAC)}

Samples were melted and methylated by the potassium hydroxide method (AOCS, method $\mathrm{Ce} 2$ ) and analyzed by GC as described before [14].

\section{Diglyceride Content (DAG)}

DAG content was analyzed on a HP narrow-bore silica column $(\mathrm{L}=10 \mathrm{~cm}$, i.d. $=2.1 \mathrm{~mm}$, particle size $=5 \mu \mathrm{m}$; Hewlett Packard, Waldron, Germany). A binary solvent system of heptane and heptane/tetrahydrofurane/acetic acid $(80 / 20 / 1, v / v / v)$ was used. Samples were dissolved in heptane $(5 \mathrm{mg} / \mathrm{mL})$ and $20 \mu \mathrm{L}$ aliquots were injected. Dipalmitin was used as an external standard and calibration curves were established to quantify the amount of DAG in the samples. The concentration of DAG was expressed as the weight percentage of the sample.

\section{Free Fatty Acid Content (FFA)}

FFA content was determined by the AOCS official method Ca 5a-40 (1993).

\section{Solid Fat Content (SFC)}

SFC was measured by Minispec mq 20 NMR analyzer (Bruker, Germany) according to the AOCS serial measurement method (Cd 16b-93, 1993) at measurement temperatures of $5,15,25,30,35$, and $40{ }^{\circ} \mathrm{C}$.

All the measurements were made in triplicates. Statistical analysis was performed by t-test $(\mathrm{p}<0.05)$.

\section{RESULTS AND DISCUSSION}

\section{Practical Control of Water Content}

Water content is a common issue for such microaqueous reaction systems. The effect of water content on the Lipozyme TL IM-catalyzed interesterification has been evaluated before [2]. It seems that the water content did not affect the enzyme function in initial reactions as well as in reuses. Reducing the water content from approx. $5 \%$ in the commercial 
preparation to $3 \%$ did not cause any significant effects on the reaction. Based on that conclusion, the water content is certainly better to be reduced further in order to minimize the formation of by-products such as FFA and DAG from hydrolysis. Taking the commercial lipase preparation for granted, it is obviously necessary to know how this water situation affects the products and process technology in actual uses. Interesterification was carried out at different enzyme dosages (Fig. 2). It can be seen that more enzyme brought more water into the reaction system so as to generate more DAG and FFA. The degree of hydrolysis has more or less a linear relationship with the enzyme dosage based on the first batch study (Fig. 2).

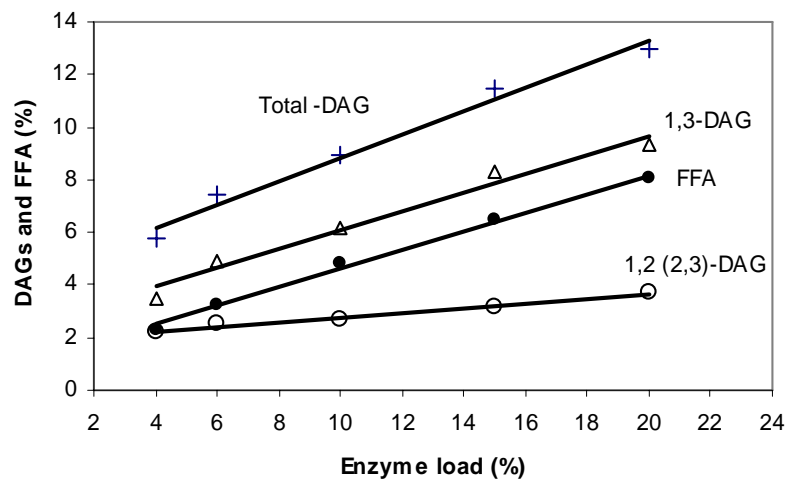

Fig. (2). Effect of enzyme load on FFA and DAG contents for the Lipozyme TL IM - catalyzed interesterification between palm stearin and coconut oil $(75 / 25, \mathrm{w} / \mathrm{w})$ in a batch reactor. Reaction conditions: temperature $60{ }^{\circ} \mathrm{C}$, stirring $700 \mathrm{rpm}$, and reaction time $6 \mathrm{~h}$.

Lipase might have two different forms of water, which are the bound water and the free water [14]. The bound water maintains the lipase activity and the free water generates byproducts during the hydrolysis process. Therefore, it is obvious that this free water in the lipase preparation should be reduced as much as possible.

Water content control in enzymes can be different for different enzymes. Water in the lipase could be controlled by adjusting the water activity with different saturated salt solutions or by adding molecular sieves to the system. For the former method, it takes too long to reach the moisture equilibrium. For the latter, even by adding $20 \mathrm{wt} \%$ molecular sieves, only $0.9 \%$ FFA was reduced in the product [15]. Both methods can only be used in laboratories, and are not practical for industrial applications.

In our early study concerning enzyme reuses for both Lipozyme RM IM and Lipozyme TL IM [2, 14], we found that the hydrolysis could be controlled by reuses of lipases. FFA decreased from 5.8 to $0.8 \%$ after $3-4$ reuses, since, most likely, the free water in the lipase was consumed during the reuses. The hydrolysis normally reaches equilibrium in 30 min. Therefore, the water removal can be done by 3-4 repeated reaction batches within one hour reaction time, using a liquid oil for example. The oil can be reused from such a lipase pre-treatment so that it will not bring higher cost in comparison with the drying of enzyme. It should be mentioned that the reuse or lipase pre-treatment gave different enzyme performance for different lipases. The activity of
Lipozyme TL IM was demonstrated to be stable during the operation. In a trial by adding certain extra water following the lipase pre-treatment procedure did not increase any activity [2], while, in such operations, Lipozyme RM IM functioned differently, demonstrating a decline of activity following the lipase pre-treatment procedure and an increase of activity after adding extra water afterwards [14]. We assume the performance of Lipozyme TL IM is due to the silica carrier function, which could have stronger partition to sufficient water for the maintenance of lipase activity during the lipase pre-treatment procedure. Under such operation, FFA content in the products can be reduced to $0.8-1.5 \%$, depending on the water content in substrate oils.

\section{Effects of Hydrolyzed Products (FFA and DAG) on Physical or Chemical Properties}

As operated with the above lipase pre-treatment procedure, FFA decreased to $1.5 \%$ for the lipase-catalyzed interesterification. Accordingly, DAG content will be also reduced but exist in the products. Certainly those by-products may affect the evaluation of physical and chemical properties of the products. The effects of hydrolyzed products were further studied by firstly removing FFA (-FFA), and then DAG (-(FFA+DAG)) through short path distillation and silica gel absorption, respectively (Scheme 1).

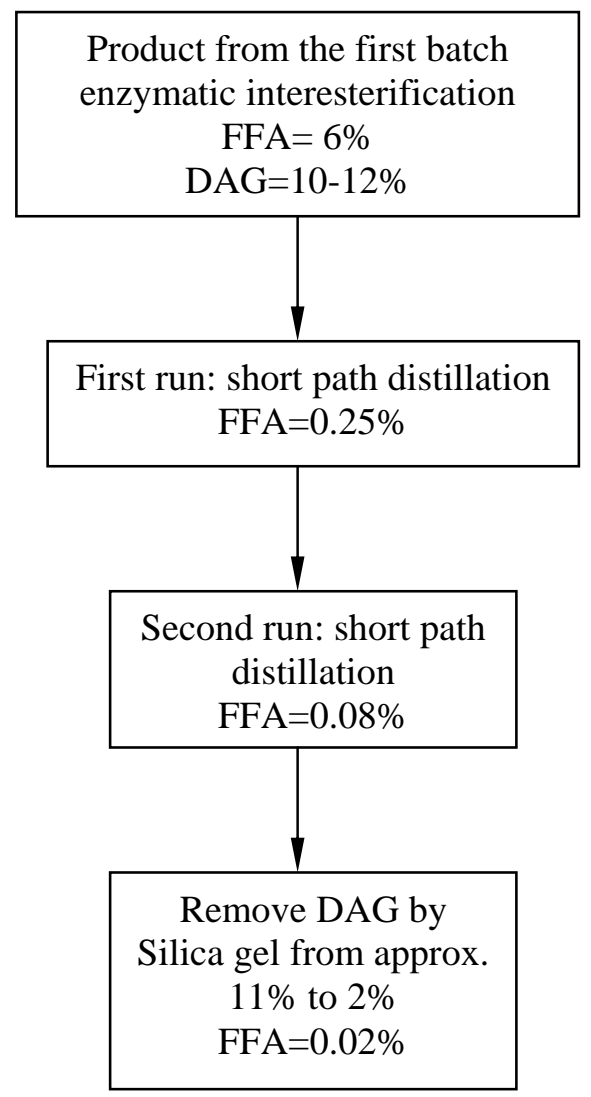

Scheme 1. Process scheme for removal of FFA and DAG in the products using short path distillation and silica gel absorption.

It can be seen that SFC of the interesterified products from the first batch was lower than the SFC of products produced with the pre-treated lipase preparation or with FFA removed (-FFA) by short path distillation from the first batch production (Table 1). The presence of FFA is assumed to have softening effect on products according to SFC meas- 
urement since the first batch reaction generated more FFA (6\%). FFA influenced not only the physical properties, but also the chemical properties. FFA is oxidized more easily than TAG. Although a small amount of FFA in fats or oils does not have marked effect on oxidative stability (FFA < $0.03 \%$ for the RBD oil), the presence of a relatively large amount of FFA facilitates incorporation of catalytic trace metals from equipment or storage tank, and thereby increases the rate of lipid oxidation [16]. Therefore, it is important to remove FFA from the products for many reasons.
On the other hand, the effects of DAG for the interesterified products did not have large impact on SFC values, except for SFC measured at $30{ }^{\circ} \mathrm{C}$ after $3 \mathrm{~h}$ reaction, in which a slightly hardening effect was found between the interesterified products treated by removing FFA and produced with the pretreated lipase preparation, as the two procedures will lead to different contents of DAG (Table $\mathbf{1}$ ).

The effects of FFA and DAG on the storage stability (Table 2) were evaluated using the products produced by the commercial lipase preparation without lipase pre-treatment

Table 1. Effect of FFA on SFC for Enzymatically Interesterified Products with "Fresh" or Pre-Treated Lipase and FFA was Removed by Short Path Distillation (Scheme 1) at Different Reaction Times in a Batch Reactor. Reaction Conditions: Blend of Fully Hydrogenated Cottonseed Oil and Soybean Oil (FHCO/SO, 1/1); 10 wt\% Lipozyme TL IM; Temperature $70{ }^{\circ} \mathrm{C}$

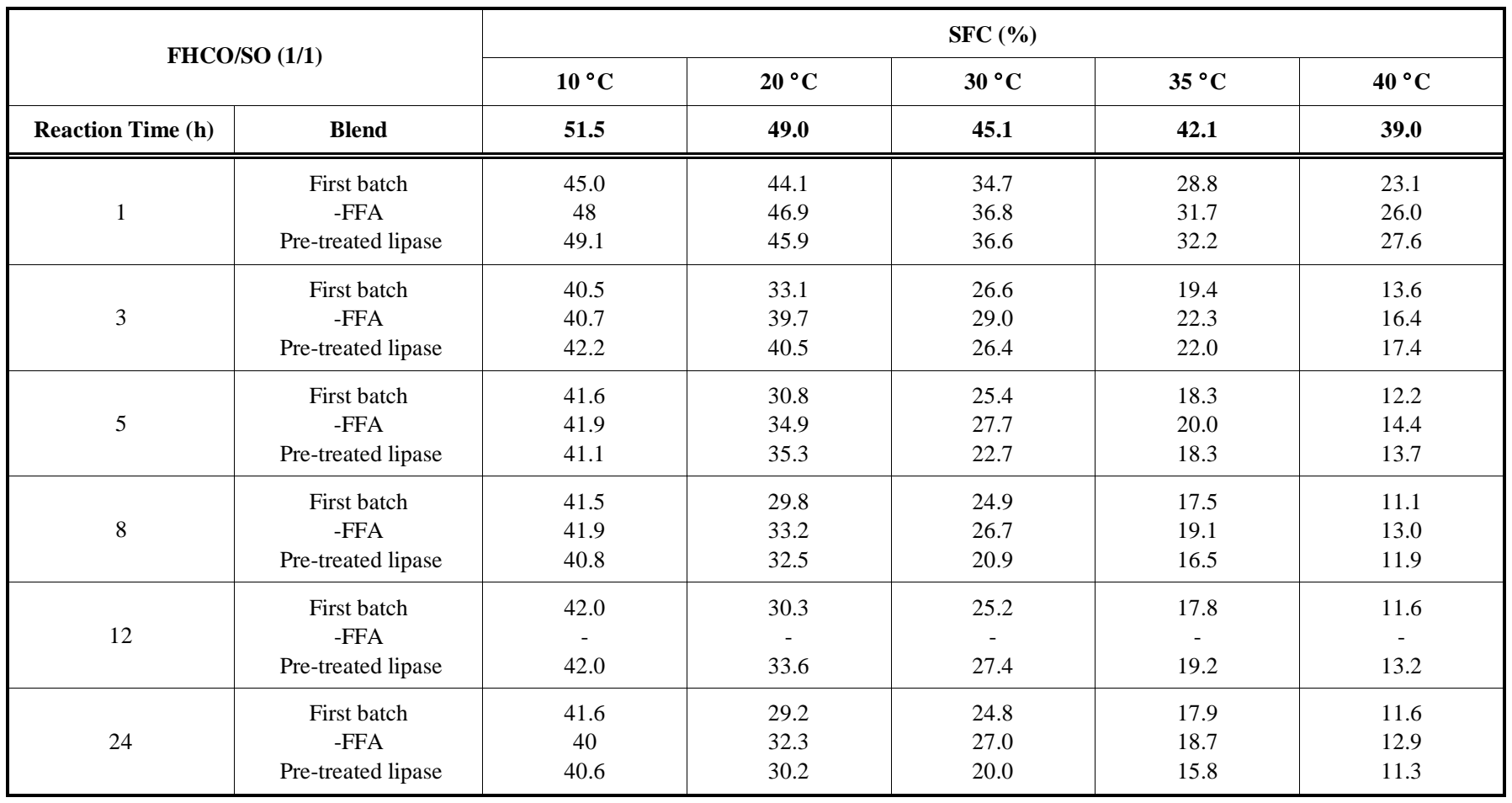

-FFA: removing FFA by short path distillation.

Table 2. Effects of FFA and DAG on the Crystal Transformation for the Products Produced at the First Batch and their Products were Further Treated with SPD and Silica Gel Absorption for Removing FFA and DAG, Respectively (Scheme 1). Reaction Conditions see Table 1

\begin{tabular}{|c|c|c|c|c|}
\hline \multirow{2}{*}{$\begin{array}{c}\text { Reaction Time } \\
(\mathbf{h})\end{array}$} & Samples & \multirow{2}{*}{ First Day } & \multicolumn{2}{|c|}{$\boldsymbol{\beta}^{\prime}$ Content (\%) } \\
\cline { 4 - 5 } & & & One Week & One Month \\
\hline \hline 0 & Blend & $\alpha+\beta^{\prime}(\beta=10 \%)$ & 50 & 44 \\
\hline \multirow{2}{*}{3} & First batch & $\alpha$ & 100 & 100 \\
& -FFA & $\alpha$ & 100 & 100 \\
& -(FFA+DAG) & $\alpha$ & 20 & 20 \\
\hline \multirow{3}{*}{8} & First batch & $\alpha$ & 100 & 90 \\
& -FFA & $\alpha$ & 100 & 20 \\
& -(FFA+DAG) & $\alpha$ & 25 & 71 \\
& Product & $\alpha$ & 85 & 85 \\
\hline
\end{tabular}

-FFA: product removed FFA; -(FFA+DAG): product removed both FFA and DAG. 
and their hydrolyzed products that were further removed by SPD and silica gel absorption (Scheme 1) at different reaction times. FFA did not show large influence on crystal transformation. However, DAG showed significant effect on the crystal transformation. There were only $20-35 \%$ of $\beta$ ' crystal left after removing DAG from the interesterified products for both one week and one month storages, meaning that most of them had changed into $\beta$ crystals. In this aspect, DAG significantly delayed the crystal transformation from $\beta$ ' to $\beta$ during the storage. This agrees to the study of Hernqvist and Anjou [17], where DAG was added into a blend of partially hydrogenated rapeseed oil and soybean oil. They reported that, after an addition of 1 to $5 \%$ of DAG in the blend, the time for developing into the $\beta$-form was prolonged to 2-3 fold. By adding 5\% DAG, the development of $\beta$-form could be delayed from 4 to 44 weeks. The presence of $3-6 \%$ of mono- and di-glycerides was treated as crystallization retarders [18].

In our study, the content of $\beta$ ' can be stabilized 2-5 times longer at a DAG content of $10-12 \%$ for the products from different reaction times. The effects of DAG on the crystal transformation decreased with the increase of interesterification degree, indicating that the triglyceride structure after greater extent of interesterification had a stronger determination on crystal transformation.

After one month storage, $44 \% \beta$ ' were left for the blend, while for the interesterified products from 3 , 8, and 24 h reactions, 100,90 , and $71 \% \beta$ ' crystals remained, respectively, after one month storage, meaning reaction and reaction degree meant something for the crystal transformation. FFA also had some effects for the $24 \mathrm{~h}$ reaction products, meaning that the effects are a multiple interaction process. Since FFA has effects also on taste and oxidation, they should be reduced anyway through either the reaction step or the refining steps.

In general, DAG has significant effect on crystal transformation, even though in a positive way concerning the use of products for margarine production, where $\beta$ ' crystals are required. However, in process development, the minimization of by-products formation is still the aim since FFA increases fat loss as well as low quality. Furthermore, DAG can give effects on chemical properties of the products.

DAG can cause acyl migration on the glycerol backbone, so that the sn-1,3 lipase specificity is lost and consequently leads to producing non-specific products. It was a very crucial problem for the production of cocoa butter substitutes or specific structured lipids $[19,20]$. Therefore, it is worthwhile to discuss the mechanisms of the reactions and acyl migration for the Lipozyme TL IM-catalyzed interesterification.

During the enzymatic interesterification, an acyl-enzyme complex and a DAG are formed at the first step of the reaction. DAG is then a key intermediate in the following reaction, since esterification step is followed by incorporating a FFA into DAG and forming a new TAG. If no acylmigration occurs, only 1,2(2,3)-DAG and FFA $\left(\mathrm{R}_{1}\right.$ and $\left.\mathrm{R}_{3}\right)$ should be generated by a sn-1,3 specific lipase; whereas with a non-specific lipase, a mixture with an even distribution of 1,3-DAG, 1,2- DAG, 2,3-DAG, together with FFA $\left(\mathrm{R}_{1}, \mathrm{R}_{2}\right.$, and $\mathrm{R}_{3}$ ) can be produced (Fig. 3 ).

In the presence of $1,2(2,3)-D A G$, acyl migration will take place and lead to the formation of 1,3-DAG, which is more stable due to the steric reason [21]. This makes difficult for 1,3-DAG to react with acyl-enzyme complex. Acyl migration is therefore a potential source of elevating DAG levels in the reaction mixture at the end point [19]. However, the migration of an acyl group on the sn- 1 or 3 position to the sn-2 position may also take place for the $1,3-\mathrm{DAG}$. The fatty acid composition at the sn-2 position is, therefore, changed due to acyl migration.

Fig. (4) shows that 1,2 (2,3)-DAG reached the equilibrium quickly (less than $1 \mathrm{~h}$ ); while 1,3-DAG increased steadily, especially at the beginning of reaction. The increase of total DAG in the system was primarily due to the increase of 1,3-DAG. This agrees to the observation in an acidolysis system for the production of cocoa-butter-substitute [19]. Of course, 1,3-DAGs could also be directly produced with a non-specific lipase. The ratio between 1,3-DAG and $1,2(2,3)$-DAG should be $1 / 2$ in such a case, if no acyl migration took place (Fig. 3). Fig. (4) showed a higher content of 1,3-DAG than 1,2(2,3)-DAG. When the system reached the equilibrium, a constant ratio of $2 / 1$ was obtained between 1,3 and 1,2-(2,3) DAG (Fig. 4). Thus acyl migration obviously existed.

Overall in the experimental performance, however, the sn-2 fatty acid composition was quite similar for both reactions with the original commercial lipase (Fig. 5A) and the pre-treated lipase (Fig. 5B). Higher water content in the system for the former, which led to a higher content of DAG,
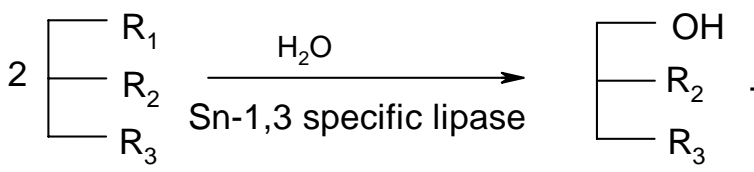

2,3-DAG
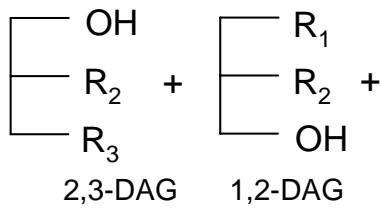

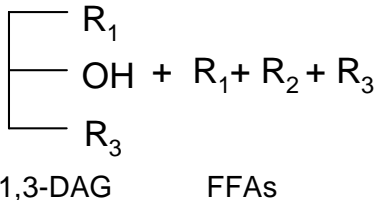

Fig. (3). Hydrolysis of TAG by sn-1,3 specific lipase or non-specific lipase. 


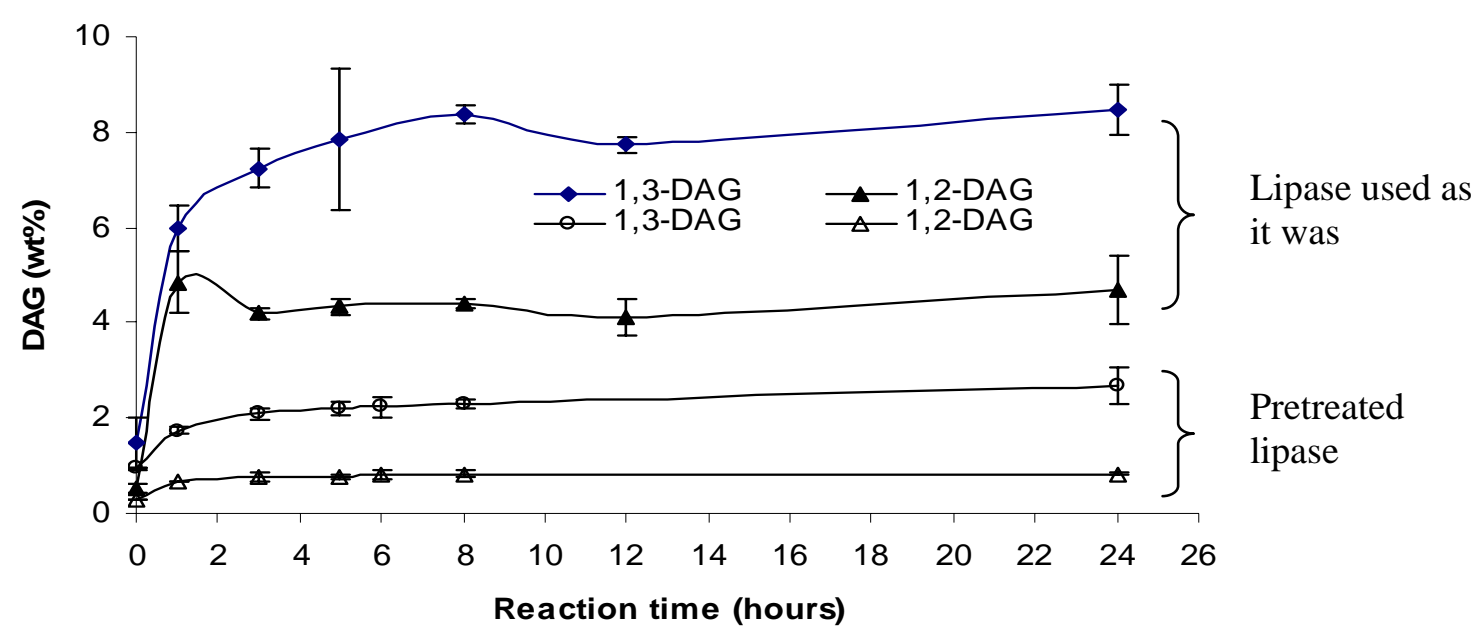

Fig. (4). Effects of reaction time and operation procedure on DAG contents in the products from lipase-catalyzed interesterification in a batch reactor. Reaction conditions see Table $\mathbf{1}$.
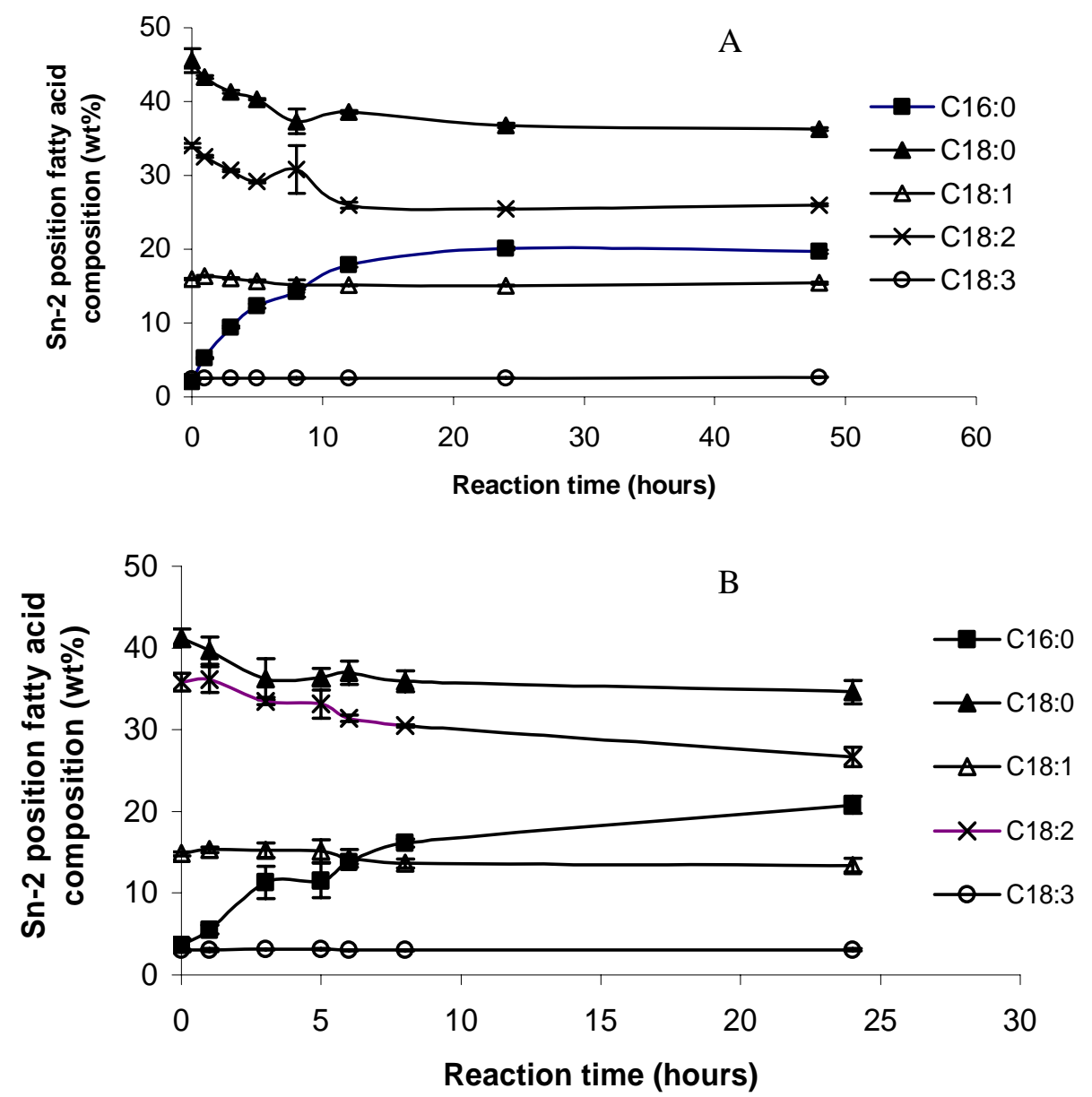

Fig. (5). Distribution of fatty acid composition at sn-2 position for lipase-catalyzed interesterification with the commercial lipase preparation without pre-tretment (A) and with pre-treatment (B). Reaction conditions see Table $\mathbf{1}$.

did not result in higher acyl migration. This might be due to the different polarities in the systems, where the water in the system decreased nucleophilicity of the lone pair for the free hydroxyl oxygen. Consequently acyl migration could be inhibited by the increase of polarity in the system. Thus, water in the system might play double roles for the acyl migration, both promoting the migration through higher DAG formation and inhibiting the migration through nucleophilicity partition. The effect of polarity on the acyl migration was also observed in a solvent system where Lipozyme RM IM showed higher sn-1,3 specificity in diethyl ether and less specificity in hexane [22].

Fig. (5) shows the fatty acid compositions at the sn-2 position at different reaction times. Palmitic acid content increased, while linoleic or stearic acid content decreased 
with the increase of reaction time. After $24 \mathrm{~h}$, the fatty acid distribution at the sn-2 position became randomized. That means that the reaction time was an important factor for the control of acyl migration. When high enzyme dosage (50\% Lipozyme RM IM) was used for the interesterification of ethyl palmitate with triolein to produce cocoa butter substitutes [19], the endpoint was reached in 13 min without forming tripalmitate even with high DAG (17\%) content in the system. This also implies that the use of packed-bed reactor could have benefit both for improving the operation efficiency and maintaining the lipase specificity, since the highest ratio of lipase to substrate is used, and, consequently, the shortest reaction time is used.

\section{Temperature Effect on Reaction Rate}

Temperature is another commonly known issue for enzymatic reactions. The temperature optimum has been evaluated for the Lipozyme TL IM-catalyzed interesterification [2]. Temperature has also double functions in the system, where enzyme activity and viscosity can be both affected. Fig. (6) shows the temperature effect on the reaction rate k, which was monitored by SFC changes measured at $40{ }^{\circ} \mathrm{C}$. Increasing temperature accelerated the reaction rate. $\mathrm{Q}_{10}$ is often used in biology and defined as the increase in reaction rate for a $10{ }^{\circ} \mathrm{C}$ interval in temperature. $\mathrm{Q}_{10}$ falls within the range of 2-3 for most chemical and enzymatic reactions. It has the following relationship: $\Delta \log$ rate $=\frac{\Delta T}{10} \log Q_{10}$ [23]. For Lipozyme TL IM-catalyzed interesterification, $\mathrm{Q}_{10}$ was about 1.3 at a temperature range from 70 to $90{ }^{\circ} \mathrm{C}$. In general, it is lower than reactions in aqueous [23] or solvent [24] media. The limitation of the mass transfer in the oil system, which has higher viscosity, might be the reason for the lower $\mathrm{Q}_{10}$ factor than in other systems. From this aspect, the further increase of temperature from 70 to $90^{\circ} \mathrm{C}$ will not provide a dramatic benefit of reaction rate increase considering a low value of $\mathrm{Q}_{10}$.

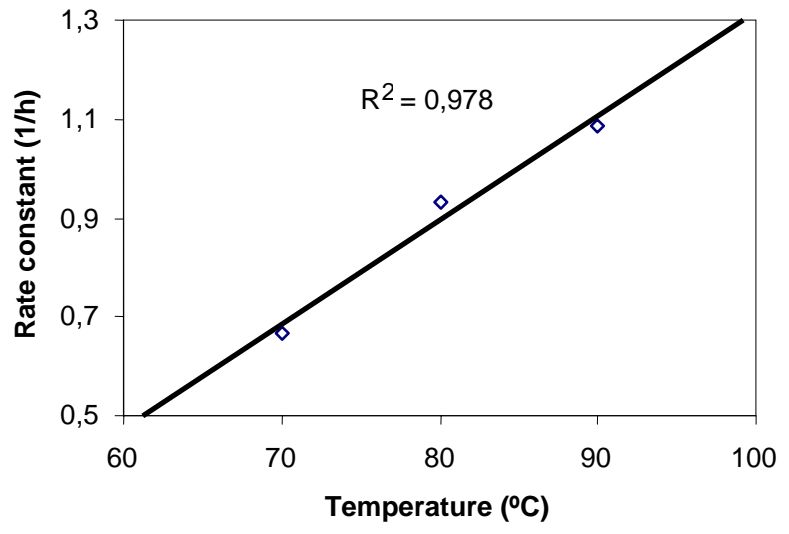

Fig. (6). Temperature effect on the reaction rate $k$ based on SFC for lipase-catalyzed interesterification in a batch reaction. Reaction conditions: blend PS/CO (70/30); lipase dosage $4 \mathrm{wt} \%$; temperature $70{ }^{\circ} \mathrm{C}$; reaction time $2 \mathrm{~h}$.

Too high temperature will also denature the structure of enzyme. Being the protein, the integrity of the three dimen- sional structure of the enzyme active site is essential for the maintenance of activity. Thus, any factors influencing the integrity of the secondary, tertiary, and quaternary structures of an enzyme will affect its activity. Temperature is one of the important factors to affect the lipase stability. At high temperatures, denaturation will be more pronounced in principle [24]. Shorter stability of the enzyme will also be expected at higher temperatures. With all these considerations, the lipase-catalyzed interesterification is suggested to run at $70{ }^{\circ} \mathrm{C}$.

\section{Feedstock Quality}

Feedstock quality is one of the issues that affect the lipase activity and stability during the operation. This again has double meaning for the process operation. There is no doubt that better quality of the feedstock will be good for the enzymatic reactions. On the other hand, processing cost has to be added to provide a higher quality of feedstock. Therefore, an evaluation was needed to visualize the effects of material quality. Fig. (7) shows the effect of oil quality on the reaction in the batch reactor. The non-deodorized blend delayed the reaction progress during the process. In another study, higher decrease (43\%) in conversion degree at $2 \mathrm{~h}$ was observed by adding a large amount of PC (7 wt\%) into the reaction system. This indicates that the impurities in the oils and fats do have strong impact on the process efficiency.

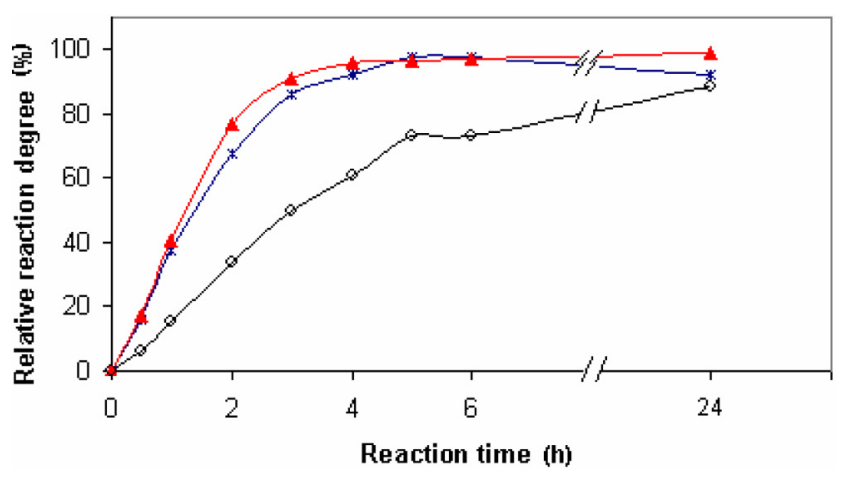

Fig. (7). Effects of impurities in the oil on the conversion degree for lipase-catalyzed interesterification in a batch reactor $(*$, nondeodorized soybean oil (SO)/coconut oil (CO) (70/30); $\boldsymbol{\Delta}$, deodorized SO/CO (70/30); o, 7\% phosphatidylcholine (PC) in the deodorized SO/CO (70/30)).

In a previous study [25], high concentration of phospholipids ( $\geq 0.5 \%$ ) was observed to cause reduction in the initial reaction rate in a solvent system for Lipozyme RM IMcatalyzed acidolysis. When experiments were conducted in 10 batches at PC concentration of $1 \%$, the largest decrease of incorporation of caprylic acid into triolein was about $40 \%$. The reduction of reaction rate is mainly due to the contact competition with the enzyme between the substrates and PC. The effects of coating and chemically binding to the active site of enzyme might be another reason for the deactivation of the enzyme in a long-term operation. Other minor components related to oil quality are also very important for the lipase activity. It was observed that a high purity of feedstock had better performance to maintain the enzyme activity by using high purity of shea oleine instead of shea oil [26]. The oil with high peroxide value (PV) decreased initial reac- 
tion rate for the glycerolysis [27]. However, it was found in another study that the initial activity of the enzyme in an acidolysis reaction was not significantly reduced even with a feedstock having a PV of $50 \mathrm{meq} / \mathrm{kg}$ in the first batch. Operation stability was reduced on the other hand. After eight runs, $50 \%$ loss of activity was observed for Lipozyme RM IM-catalyzed reaction between triolein and lauric acid [28]. Thus, we can claim from the study that the feedstock quality is not a trivial matter for the process development. It can influence the performance of the process in a considerably large extent. Therefore, feedstock quality should be improved in general, even though an economical balance should be further evaluated for a specific operation. For the lipase-catalyzed interesterification, refined, bleached and deodorized oil is recommended to be used. In fact, chemical interesterification is stricter with the oil quality. Both FFA and water as well as others are fatal to the reaction, as they inactivate the catalyst. The contents of FFA, phosphorous, peroxide value, water and anisidine value should be less than $0.05 \%, 2 \mathrm{ppm}, 0.5 \mathrm{meq} / \mathrm{kg}, 0.05 \%$, and 10 , respectively, according to practical processes [29].

\section{CONCLUSION}

From this study, it can be seen that the water in the system, which is mainly from the lipase, has effects on both physical and chemical properties of enzymaticallyinteresterified products. A high content of water in the system leads to high content of FFA and DAG. FFA has softening effect on SFC. DAG retarded the crystal transformation. The amount of DAG due to hydrolysis was not the only reason leading to the increase of acyl migration. Reaction time was more crucial to inducing acyl migration than water content in the system. The water content can be optimized by pre-treating or pre-drying lipase in order to improve the yield. Since the temperature has only slight effect on the reaction rate compared to other systems of using enzyme as catalyst, a moderate temperature is recommended for a longterm operation in the range of 60 to $80{ }^{\circ} \mathrm{C}$. The phospholipids bind to the enzyme or to the enzyme support, and indirectly affect the enzyme activity. Therefore, it is recommended to use refined, bleached, and deodorized fats and oils in order to reduce the lipase deactivation during the operation.

\section{ACKNOWLEDGEMENTS}

Technical supports from Kristina Brun Larsen are thanked. Partial support from Ministry for Food, Agriculture, and Fishery through its Innovation Programme is acknowledged.

\section{REFERENCES}

[1] Christensen MW, Andersen L, Kirk O, Holm HC. Enzymatic interesterification of commodity oils and fats: approaching the tones scale. Lipid Technol 2001; 4: 33-7.

[2] Zhang H, Xu X, Nilsson J, Mu H, Adler-Nissen J, Høy C-E. Production of margarine fats by enzymatic interesterification with silica-granulated Thermomyces lanuginosus lipase in a large-scale study. J Am Oil Chem Soc 2001; 78: 57-64.

[3] Zhang H, Pedersen LS, Kristensen D, Adler-Nissen J, Holm HC. Modification of margarine fats by enzymatic interesterification: Evaluation of a solid-fat-content-based exponential model with two groups of oil blends. J Am Oil Chem Soc 2004; 81: 653-58.
[4] Zhang H, Smith P, Adler-Nissen J. Effects of degree of enzymatic interesterification on the physical properties of margarine fats Solid fat content, crystallization behaviour, crystal morphology and crystal network. J Agric Food Chem 2004; 52: 4423-31.

[5] Zhang H, Jacobsen C, Adler-Nissen J. Storage stability study for margarines produced by enzymatically interesterified fats compared to the margarines by the conventional methods I. Physical properties. Eur J Lipid Sci Technol 2005; 107: 530-9.

[6] Zhang H, Jacobsen C, Pedersen LS, Christensen MW, AdlerNissen J. Storage stability study for margarines produced by enzymatically interesterified fats compared to the margarines by the conventional methods I. Chemical properties. Eur J Lipid Sci Technol 2006; 108: 227-38.

[7] Ronne T, Pedersen L, Xu X. Triglyceride selectivity of immobilized Thermomyces lanuginosa lipase in interesterification. J Am Oil Chem Soc 2005; 82 (10): 737-43.

[8] Ronne T, Yang T, Mu H, Jacobsen C, Xu X. Enzymatic interesterification of butterfat with rapeseed oil in a continuous packed bed reactor. J Agric Food Chem 2005; 53 (14): 5617-24.

[9] Chang T, Lai X, Zhang H, Sondergaard I, Xu X. Monitoring lipasecatalyzed interesterification for bulky fat modification with FTIR/NIR spectroscopy. J Agric Food Chem 2005; 53(26): 9841-7.

[10] Zhang $\mathrm{H}, \mathrm{Mu} \mathrm{HL}$, Xu X. Monitoring lipase-catalyzed butterfat interesterification with rapeseed oil by Fourier transform nearinfrared spectroscopy. Anal Bioanal Chem 2006; 386 (6): 1889-97.

[11] Osorio NM, da Fonseca MMR, Ferreira-Dias S. Operational stability of Thermomyces lanuginosa lipase during interesterification of fat in continuous packed-bed reactors. Eur J Lipid Sci Technol 2006; 108(7): 545-53.

[12] Siew WL, Cheah KY, Tang WL. Physical properties of lipasecatalyzed interesterification of palm stearin with canola oil blends. Eur J Lipid Sci Technol 2007; 2: 97-106.

[13] Reddy SY, Prabhakar JV. Study on the polymorphism of normal triglyverides of sal (shorea robusta) fat by DSC 1. Effect of diglycerides. J Am Oil Chem Soc 1986; 63: 672-6.

[14] Zhang H, Xu X, Mu H, Nilsson J, Adler-Nissen J, Høy C-E. Lipozyme IM-catalyzed interesterification for the production of margarine fats in a $1 \mathrm{~kg}$ scale stirred tank reactor. Eur J Lipid Sci Technol 2000; 102: 411-8.

[15] Zainal Z, Yusoff MSA. Enzymatic interesterification of palm stearin and palm kernel olein. J Am Oil Chem Soc 2002; 76: 10038.

[16] Nawar WW. Lipids, in Food Chemistry, edited by Fennema OR, Marcel Dekker, Inc., New York 1996; pp. 225-320.

[17] Hernqvist L, Anjou K. Diglycerides as a stabilizer of the $\beta$ '-crystal form in margarines and fats. Fett/Lipid 1983; 85: 64-6.

[18] Hoffmann G. Production of edible-fat products of high fat content, in The chemistry and technology of edible oils and fats and their high fat products. Academic Press, London, 1989; pp. 279-338.

[19] Bloomer S, Adlercreutz P, Mattiasson B. Triglyceride interesterification by lipases 2 . Reaction parameters for the reduction of trisaturated impurities and diglycerides in batch reactions. Biocatalysis 1991; 5 : 145-62.

[20] X Xu, Balchen S, Høy C-E, Adler-Nissen J. Pilot batch production of specific-structure lipids by lipase-catalyzed interesterification: preliminary study on incorporation and acyl migration. J Am Oil Chem Soc 1998; 75: 301-8.

[21] Kodali DR, Tercyak A, Fahey DA, Small DM. Acyl migration in 1,2-diapamitoyl - sn - glycerol. Chem Phy Lipids 1990; 52: 16370 .

[22] Goh SH, Yeong SK, Wang CW. Transesterification of cocoa butter by fungal lipases: Effect of solvent on 1,3-specificity. J Am Oil Chem Soc 1993; 70: 567-70.

[23] Whitaker JR. Effect of temperature on enzyme-catalyzed reactions, in Principles of Enzymology for the Food Sciences, edited by Whitaker JR. Marcel Dekker, Inc., New York 1972; pp.319-49.

[24] Egger D, Wehtje E, Adlercreutz P. Characterization and optimization of phospholipase $\mathrm{A}_{2}$ catalyzed synthesis of phosphatidylcholine, BBA-Protein. Struct Mol Enzymol 1997; 1343: 76-84.

[25] Wang YQ, Gordon MH. Effect of lipid oxidation products on the transesterification activity of an immobilized lipase. J Agric Food Chem 1991A; 39: 1693-5. 
[26] Wisdom RA, Dunnill P, Lilly MD. Enzymatic interesterification of fats: laboratory and pilot-scale studies with immobilized lipase from Rhizopus arrhizus. Biotechnol Bioeng 1987; 29: 1081-5.

[27] Ohta Y, Yamane T, Shimizu S. Inhibition and inactivation of lipase by fat peroxide in the course of batch and continuous glycerolysis of fat by lipase.. Agric Biol Chem 1989; 53: 1885-90.
[28] Wang YQ, Gordon MH. Effect of phospholipids on enzymecatalyzed transesterification of oils. J Am Oil Chem Soc 1991B; 68: 588-90.

[29] Kellens M. Oil modification processes, in Edible Oil Processing, edited by Hamm W, Hamilton RJ, Sheffield Academic Press, Sheffield 2000; pp. 129-73. 